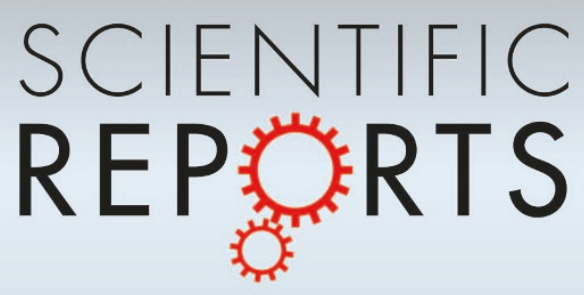

OPEN

SUBJECT AREAS:

NATURAL PRODUCTS

COMPUTATIONAL MODELS

Received

30 January 2014

Accepted

1 April 2014

Published

6 May 2014

Correspondence and requests for materials should be addressed to J.S. (shangiing21cn@ 163.com) or F.-R.Y. (f.r.yan@163. com)

* These authors contributed equally to this work.

\section{Lipid modulatory activities of Cichorium glandulosum Boiss et Huet are mediated by multiple components within hepatocytes}

\author{
Lin Ding ${ }^{1 *}$, Jun-Lin Liv ${ }^{2 *}$, Waseem Hassan' ${ }^{1}$ Lu-Lu Wang ${ }^{1}$, Fang-Rong Yan ${ }^{2} \&$ Jing Shang ${ }^{1}$
}

\begin{abstract}
'National Center for Drug Screening \& State Key Laboratory of Natural Medicines, China Pharmaceutical University, Jiangsu Province, 210009 , P. R. China, ${ }^{2}$ Research Center of Biostatistics and Computational Pharmacy, China Pharmaceutical University, Jiangsu Province, 210009 , P. R. China.
\end{abstract}

To investigate a possible methodology of exploiting herbal medicine and design polytherapy for the treatment of non-alcoholic fatty liver disease (NAFLD), we have made use of Cichorium glandulosum Boiss et Huet (CG), a traditional Chinese herbal medicine that has been proven to be effective in treating hepatic diseases. Here, we report that the extract of CG effectively reduced lipid accumulation under conditions of lipid overloading in vivo and in vitro (in a rat high-fat diet model and a hepG2 cell model of free fatty acid treatment). CG extract also protected hepatocytes from injury and inflammation to aid its lipid-lowering properties (in a rat high-fat diet model and a L02 cell model of acetaminophen treatment). Serum chemistry analysis accompanied by in vitro drug screening confirmed that CG-4, CG-10 and CG-14 are the lipo-effective components of CG. Western blotting analysis revealed that these components can regulate key lipid targets at the molecular level, including CD36, FATP5 and PPAR-a, thus the lipid oxidation and lipid absorption pathways. Finally, we adopted the experimental design and statistical method to calculate the best combination proportion (CG-4: CG-10: CG-14=2.065: 1.782: 2.153) to optimize its therapeutic effect.

M ulti-component therapy and the incorporation of components from various plant sources with related medicinal properties is the cornerstone of traditional medical practices. This particular system has been in place for centuries in China in a distinctive form, with a broad range of healing benefits. Without doubt, multi-component therapy offers the advantage of synergism at the expense of a great deal of complexity and difficulty in understanding and studying the mechanism of their activities; which has hampered the modernization drive of traditional Chinese medicines (TCMs).

Non-alcoholic fatty liver disease (NAFLD) is the hepatic expression of metabolic syndrome (MS), and its prevalence is expanding at an alarming rate worldwide ${ }^{1}$. Clinically, NAFLD is characterized by an accumulation of triglycerides accompanied by a roughly $5 \%$ increase in liver weight due to lipid storage ${ }^{2,3}$, and affected liver tissues generally show slight lobular inflammation and raised alanine transaminase (ALT) levels ${ }^{4,5}$. Lipid storage within hepatocytes is the hallmark of NAFLD and is the key factor for its progression into succeeding inflammatory states and carcinomas. One important player in hepatic lipid metabolism is peroxisome proliferatoractivated receptor alpha (PPAR- $\alpha)^{6}$, which has been shown to play a role in all phases of lipid metabolism: lipogenesis, fat oxidation and lipid transport. Similarly, $\mathrm{FATP}^{7}$ is involved in fatty acid uptake and has proved to be an aggravating factor in NAFLD pathogenesis. $\mathrm{CD} 36^{8}$ is another important target that is involved in the hepatic fat storage nexus, as it is involved in the uptake and transport of lipids upstream. Upregulated CD36 has been demonstrated to be involved in hepatic lipid storage. As NAFLD involves diverse and multiple targets, its successful treatment may involve the incorporation of multi-components from natural sources.

Cichorium glandulosum Boiss et Huet (CG) is a traditional Chinese medicine distributed in the Aksu region of Xinjiang, parts of Caucasia and Turkey ${ }^{9}$. Traditionally, it has been used widely for curing various hepatic diseases and has had beneficial effects on appetite and digestion ${ }^{10}$. To date, seed extracts from CG have been reported to produce anti-oxidant effects ${ }^{11}$, while underground parts of the plant have been associated with hepatoprotective activities $^{12}$. Furthermore, many pharmacologically active constituents from the class of sequterpenoids ${ }^{13}$ and flavonoids ${ }^{14}$ have been isolated from CG, but none of the beneficial mono-components is reported to be as 
effective as the whole extract from CG. This indicates that the lipidlowering effects of CG may stem from the combination of multicomponents affecting multi-targets within cells.

In our current study, we have tested the effects of CG extract on two different and established hepatic models in vitro and have used a high fat diet (HFD) model in vivo to scientifically rationalize the pharmacological activities of CG. Furthermore, we identified three major activity responsible constituents in attenuating hepatic lipid accumulation. Hence, we attempted to ascertain the specific molecular targets responsible for their lipid-lowering response and to develop an optimum composition of the active components from CG for the maximum lipid-lowering efficacy.

Recently, numerous mathematical researchers have been working on the development of optimal drug combinations ${ }^{15,16}$. For example, Yoon et al. ${ }^{15}$ proposed a novel stochastic optimization algorithm that analyzes how the concentration change of a specific drug affects the overall drug response, thereby providing information on how the drug concentration should be adjusted to improve its response. It was shown that this enhanced search algorithm could provide an effective framework for identifying potent drug combinations that lead to optimal drug response. Wong et al. ${ }^{16}$ proposed another optimization framework based on a stochastic optimization algorithm, called the Gur Game algorithm. In this work, the closed-loop optimization approach was shown to effectively identify a potent drug mixture, without the need for detailed information about the effects of each agent on the intracellular pathways. The results of this study suggested that a much lower dosage is required of a drug mixture than individual drug components in the viral infection model. Although different types of statistical models that are effective in various disease conditions have been established, few have been linked with large-scale pharmacological experiment-based evidence. In this study, we have used the Uniform Design to obtain a set of multiple drug component combinations and tested them on a lipidinduced hepatocyte model. From the results of response surface and contour plots, we can achieve the maximum effect and optimum proportion. This is a useful step that may be utilized for future research in order to optimize the effects of multi-component drugs on intracellular pathways.

\section{Results}

Serum and liver biochemical assays. The markers for hepatic lipid accumulation can be traced in the serum. The extent of change can be used as an indicator for the extension of lipid accumulation in liver. Serum level of TC, TG, ALT, AST and LDL-c all show increase in NAFLD. As shown in Table 1, the CG extract decreased all of these parameters in a dose dependent manner. HDL-c, which is considered a favorable lipid, is increased at higher doses of CG extract.
Moreover, hepatic tissue analysis for lipid peroxidation product MDA and enzyme LDH decreased in a dose-dependent pattern. Interestingly, the clinical lipid-lowering drug Bezafibrate also produced similar results both in the serum and liver analysis (Table 1).

Liver histopathological analysis. We performed H\&E staining to take into account the relative hepatic histology and arrangement. As shown in Fig. 1a, the basal diet group rats (A) had few or no abnormal changes in their tissue histology; their cells are, in comparison, wellarranged with no apparent inflammatory cell infiltration. In the HFD group (B), the cell size increased with inflammatory cell infiltration around the central vein. In the treatment groups, the CG extract (D, E) improved the liver histology seemingly in a dose dependent $(9.6 \mathrm{~g} /$ $\mathrm{kg}$ and $4.8 \mathrm{~g} / \mathrm{kg}$ ) manner. The Bezafibrate treatment group (C) has shown comparable effects to the CG extract (Fig. 1). The Oil red staining method was applied to determine the lipid storage lever within hepatocytes (Fig. 1b). It can be observed that the CGextract treatment group showed reduced size of lipid deposits (D, E), compared to model (B), which exhibited large fat depots within cells. The effects of the CG extract can be compared with those of Bezafibrate (C).

CG extract caused fewer lipid droplets in the FFA-induced HepG2 cell model. Lipid accumulation and an increased number of lipid droplets are the hallmark of NAFLD. FFAs are important for many cellular functions and have been used to induce a well-established steatosis model ${ }^{17}$. HepG 2 cells were cultured and induced with $1 \mathrm{mM}$ FFA for 24 hours before measuring the total lipids using the Oil red staining method. As shown in Fig. 2a, CG extract greatly decreased the number of lipid droplets compared to the FFA induced group only. Observations from the oil red staining experiments were quantified by measuring the fluorescence intensity at $500 \mathrm{~nm}$. The results (Fig. 2b) confirm the Oil red $\mathrm{O}$ staining investigations.

CG extract protects L02 cells from Acetaminophen (AAP) induced injury. Acetaminophen (AAP) is used as a successful model to induce hepatic injury ${ }^{18}$. Once AAP enters the body, it can be transformed into active middle metabolites that are affected by the mixed-function oxidase of CYP450. These metabolites will then combine with liver macromolecules. If a toxic dose is taken, more toxic metabolites will be created that will lead to liver injury, elevated cell membrane permeability, increased serum lactate dehydrogenase $(\mathrm{LDH})$ content $^{19}$, decreased the glucose (GLU) consumption ${ }^{20}$ and liver necrosis. We induced L02 cells with AAP (5 mmol/L) for 24 hours to observe hepatic injury level and repairing activity of CG extract. We used Diammonium glycyrrihizinate (DG) at a

Table 1 | Biochemical analysis of serum and liver tissue parameters with and without CG treatment

\begin{tabular}{|c|c|c|c|c|c|c|}
\hline & Parameters & $\mathrm{BD}$ & HFD & Bezafibrate $(0.1 \mathrm{~g} / \mathrm{kg})$ & CG-high $(9.6 \mathrm{~g} / \mathrm{kg})$ & CG-low $(4.8 \mathrm{~g} / \mathrm{kg})$ \\
\hline & Body Weight(g) & $392.21 \pm 21.06 * *$ & $443.63 \pm 26.74$ & $433.15 \pm 24.53$ & $390.53 \pm 18.59 * *$ & $409.00 \pm 12.29 * *$ \\
\hline \multirow[t]{4}{*}{ Serum } & $\mathrm{TC}(\mathrm{mmol} / \mathrm{L})$ & $0.84 \pm 0.13^{* *}$ & $2.67 \pm 0.47$ & $1.58 \pm 0.17^{*}$ & $1.04 \pm 0.39 * *$ & $1.78 \pm 0.66$ \\
\hline & HDL-C (mmol/L) & $1.69 \pm 0.17 *$ & $1.10 \pm 0.20$ & $1.50 \pm 0.29$ & $1.49 \pm 0.12^{*}$ & $0.80 \pm 0.28$ \\
\hline & $\mathrm{LDL}-\mathrm{C}(\mathrm{mmol} / \mathrm{L})$ & $0.41 \pm 0.08 * *$ & $2.00 \pm 0.50$ & $0.50 \pm 0.30 * *$ & $0.50 \pm 0.10 * *$ & $0.80 \pm 0.30 * *$ \\
\hline & $\operatorname{ALT}(U / L)$ & $76.3 \pm 14.80 * *$ & $268.0 \pm 17.01$ & $87.6 \pm 11.12 * *$ & $74.8 \pm 9.92 * *$ & $89.3 \pm 14.75^{* *}$ \\
\hline & $\mathrm{TG}$ (mg/g liver) & $150 \pm 32.85^{*}$ & $225 \pm 29.46$ & $186 \pm 37.92 *$ & $172 \pm 50.43^{*}$ & $193 \pm 47.33^{*}$ \\
\hline & $\mathrm{MDA}(\mathrm{nmol} / \mathrm{mg})$ & $0.67 \pm 0.14^{* *}$ & $1.32 \pm 0.18$ & $0.80 \pm 0.13^{*}$ & $0.81 \pm 0.08 *$ & $0.99 \pm 0.13$ \\
\hline & $\mathrm{LDH}(\mathrm{U} /$ gprot) & $1053 \pm 161^{* *}$ & $1912 \pm 185$ & $1221 \pm 82^{* *}$ & $1333 \pm 185^{*}$ & $1588 \pm 195$ \\
\hline & $\mathrm{LD}$ (mmol/gprot) & $0.31 \pm 0.07 * *$ & $0.78 \pm 0.14$ & $0.38 \pm 0.08 *$ & $0.47 \pm 0.10 *$ & $0.57 \pm 0.05$ \\
\hline
\end{tabular}

BD: basal diet; HFD: high-fat diet; SD rats were fed with or without HFD for 9 weeks. CG extract was administered intragastrically for a specified period of time. Serum and livers were isolated as above described in the methods. The values are shown as the means \pm s.d. *symbolizes $p<0.05$; and **for $p<0.01$ vs HFD. 
a

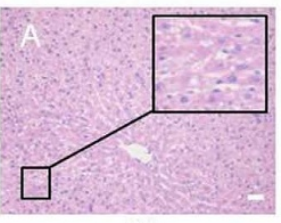

$\mathrm{BD}$

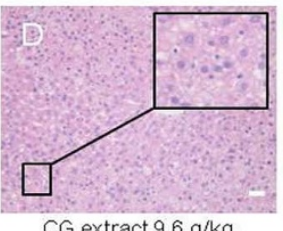

CG extract $9.6 \mathrm{~g} / \mathrm{kg}$

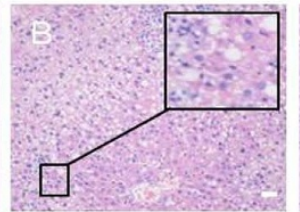

HFD

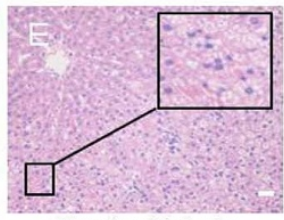

CG extract $4.8 \mathrm{~g} / \mathrm{kg}$

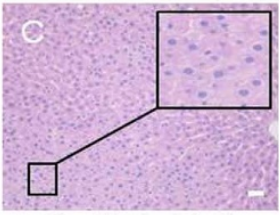

Bezafibrate $0.1 \mathrm{~g} / \mathrm{kg}$

b

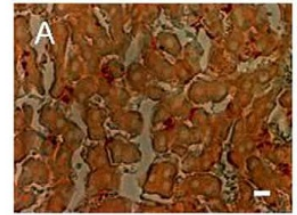

$\mathrm{BD}$

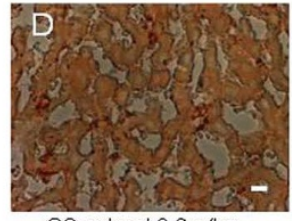

$\mathrm{CG}$ extract $9.6 \mathrm{~g} / \mathrm{kg}$

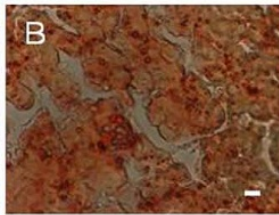

HFD

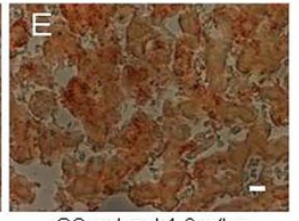

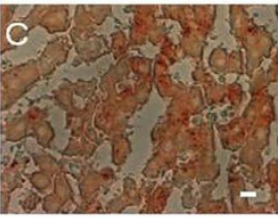

Bezafibrate $0.1 \mathrm{~g} / \mathrm{kg}$

Figure $1 \mid$ Histiopathological analysis of hepatocytes from HFD-induced SD rats. (a) Liver samples were stained with H\&E after treatment with $10 \%$ formaldehyde and embedding with paraffin. (b) Oil red O staining (small red circle) shows lipid deposits in the liver samples. The effects of H\& E staining and oil red staining can be observed in different treatment groups; The SD rats were divided into (A) the basal diet group (B) the HFD group (C) the HFD with Bezafibrate (0.1 g/kg/day) group (D) the HFD with high dose CG extract ( $9.6 \mathrm{~g} / \mathrm{kg} /$ day) group and (E) the low dose CG extract $(4.8 \mathrm{~g} / \mathrm{kg} /$ day) group. Scale bars $=800 \mu \mathrm{m}$ (figure $1 \mathrm{a}) ; 100 \mu \mathrm{m}$ (figure $1 \mathrm{~b}$ ).

concentration of $0.2 \mathrm{mg} / \mathrm{mL}$, which is commonly used in clinical medicine to reverse liver injury, as a control to compare the effectiveness of CG extract in hepatotoxicity. Our morphological observations showed that the AAP induced L02 cells demonstrated cell shrinkage compared with the control group (Fig. 3a). As expected, both the DG and CG extract treatment reversed the AAP induced hepatotoxicity (Fig. 3a), and both manifested similar effects compared with the model group. The data clearly showed that $0.2 \mathrm{mg} / \mathrm{mL}$ of CG extract inhibited hepatic injury, as assessed by measurements of LDH release (Fig. 3b) and GLU consumption (Fig. 3c) compared with the model group $(\mathrm{p}<0.05)$.

HPLC-UV method for serum chemistry study. We established the HPLC-UV method for serum chemistry assay to find the composition base of the CG extract. We compared the chromatograph of the CG extract with serum taken from the CG extract high dose treatment group and serum from the BD group. We found six compounds entered the blood (CG-2, CG-3, CG-4, CG-8, CG-10, and CG-14). These compounds are indicated by downward-facing arrows (Fig. 4). The peaks of CG-2 and CG-8 did not show up in the chromatograph of the CG extract and serum from the BD group, but existed in the serum of the CG extract in the high-dose treatment group. We therefore speculate that these may represent the metabolites of the CG extract. At this stage, we hypothesized that the lipid-lowering activity of CG extract was likely due to any or all of these six compounds, and that CG-3, CG-4, CG10, CG14 are from the origin of CG. We continued our work to determine which of these compounds is responsible for fat reducing activity of CG.

Mono-Component in vitro drug screening. From the above serum chemistry study we identified four potential active components (CG-3, CG-4, CG-10 and CG-14) in the CG extract. Thus, we decided to perform in vitro drug screening on them. The four components from the CG extract were separated and extracted. We used the FFA-induced HepG2 cell high fat model to determine the effects of the four components on intracellular triglycerides (Fig. 5). Our results showed that CG-3 is less effective than CG-4, CG-10 and CG-14 on the down regulation of TG content. Subsequently, the lipid droplets were identified by Nile red staining (Fig. 6a) and quantified by Flow Cytometric analysis a
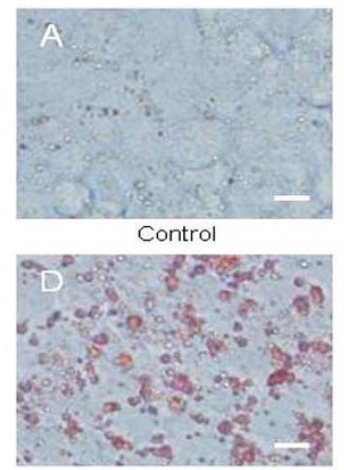

CG extract $0.2 \mathrm{mg} / \mathrm{mL}$
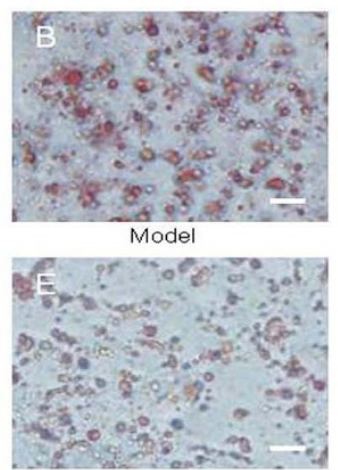

CG extract $0.1 \mathrm{mg} / \mathrm{mL}$

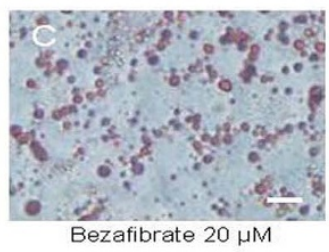

Bezafibrate $20 \mu \mathrm{M}$ b

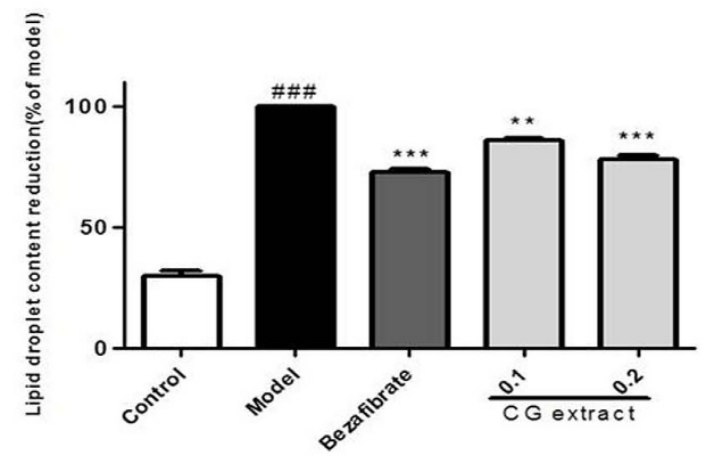

Figure $2 \mid$ The effects of CG extract on lipid droplet reduction in HepG2 cells. Hepatocytes were induced with 1 mM FFA for a specified period of time, and lipid accumulation was observed and measured using the Oil red O staining method. (a) Pictographic analysis (scale bars $=20 \mu \mathrm{m})$ after Oil red O staining. (A) The control group; (B) The FFA-induced group; (C) The FFA with Bezafibrate (20 $\mu \mathrm{M})$ group (D) The FFA with high dose CG extract $(0.2 \mathrm{mg} / \mathrm{mL})$ group (E) The FFA with low dose CG extract $(0.1 \mathrm{mg} / \mathrm{mL})$ group. (b) The contents of the lipid droplets were significantly lower in the different CG extract treatment groups than in the FFA-only treatment group. The values are shown as the means \pm s.d. and are representative of three independent experiments. **for $\mathrm{p}<0.01 ; * * *$ for $\mathrm{p}<0.001$ vs model and ${ }^{\# \# *}$ symbolizes $\mathrm{p}<0.001$ vs control. 


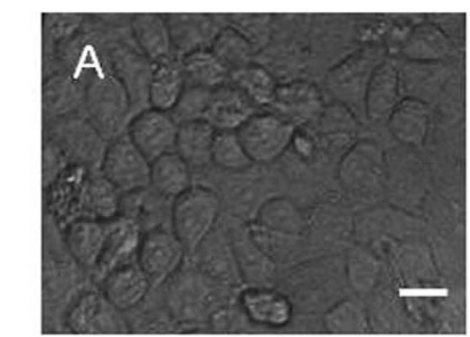

Control

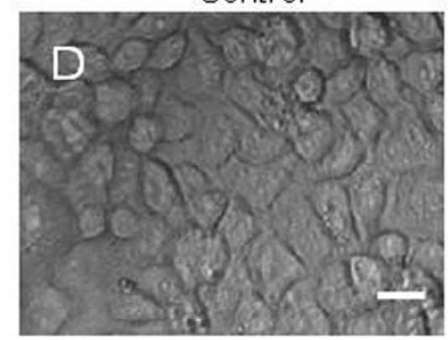

CG extract $0.2 \mathrm{mg} / \mathrm{mL}$

b

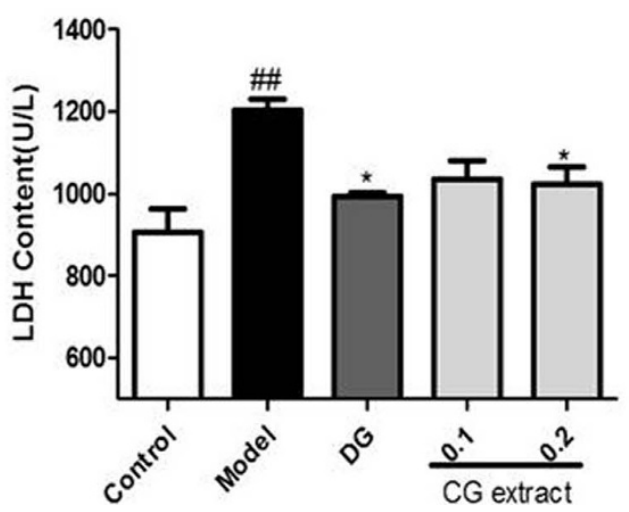

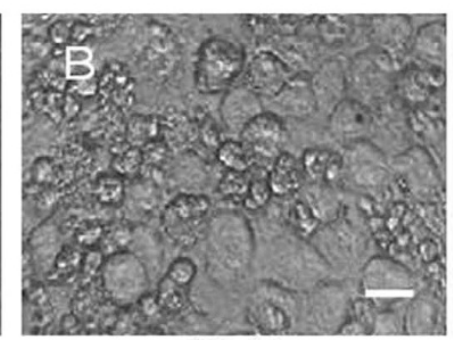

Model

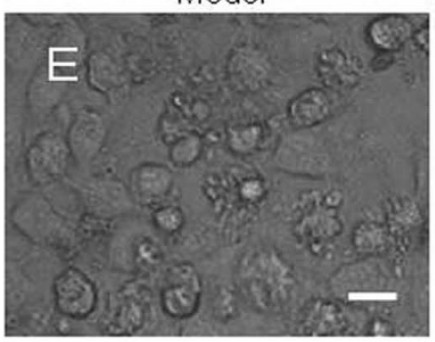

CG extract $0.1 \mathrm{mg} / \mathrm{mL}$

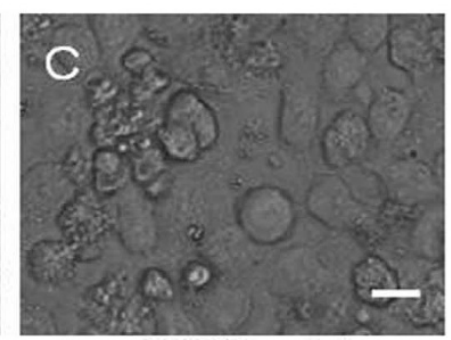

DG $0.2 \mathrm{mg} / \mathrm{mL}$

C

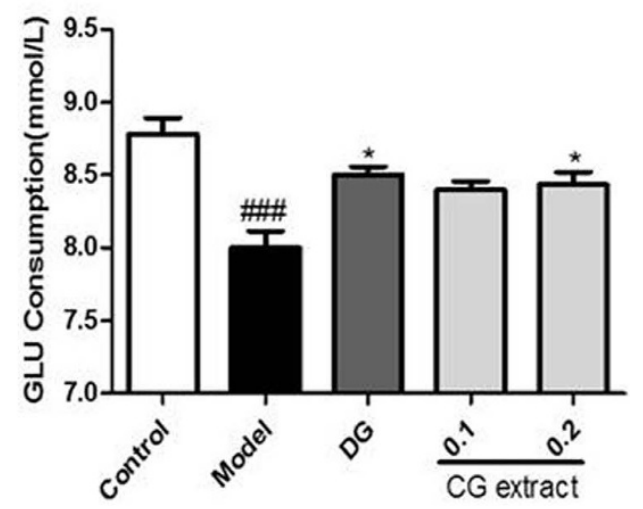

Figure 3 The morphological observation of L02 cell affected by Acetaminophen, Diammonium glycyrrihizinate and CG extract (scale bars=20 $\mu \mathrm{m}$ ). (a) L02 cells were divided into (A) Control group, (B) the AAP-only ( $5 \mathrm{mmol} / \mathrm{L}$ ) induced group, (C) the AAP-induced group co-treated with known AAP regulator Diammonium glycyrrihizinate $(0.2 \mathrm{mg} / \mathrm{mL}),(\mathrm{D})$ the AAP-induced group with high-dose CG extract $(0.2 \mathrm{mg} / \mathrm{mL})(\mathrm{E})$ the AAP-induced group with low dose CG extract $(0.1 \mathrm{mg} / \mathrm{mL}$ ). (b) LDH release and (c) GLU consumption measurement of L02 cell affected by the AAP, DG and CG extract. The values are shown as the means \pm s.d. and are representative of three independent experiments. ${ }^{*}$ for $\mathrm{p}<0.05$ vs Model and ${ }^{\# \#}$ for

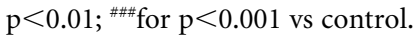

(Fig. 6b). The relative effects of CG-4, CG-10, CG-14 on HepG2 cells induced by FFA were analyzed. We intended to find the major mono-component responsible for the effect of the CG extract on lipid metabolism within hepatocytes.

Chemical structures of CG-4, CG-10 and CG-14. The characteristics of these three components (Fig. 7) have been discussed in our previous work ${ }^{21}$. The structure similarity is that they are flavanoids, and have the same quercetin moieties.

Protein expression of PPAR- $\alpha$, FATP5 and CD36. PPAR- $\alpha$ is mainly expressed in tissues with a high level of fatty acid catabolism, such as the liver, and significant data have positively linked it with lipid metabolism ${ }^{6}$. We showed here that CG-4, CG-10, and CG-14 all increase the PPAR- $\alpha$ expression in HepG2 cells and liver tissue (Fig. 8). Similarly, FATP5 and CD36 are associated with increased lipid uptake in hepatocytes ${ }^{7,8}$ and can regulate hepatic lipids negatively during the disease state. CG-4, CG-10 and CG-14 all showed a relative decrease in the protein expression of CD36
(Fig. 8) in both HepG2 cells and liver tissue. The three components also showed a down-regulation in the expression of FATP5 in HepG2 cells compared with the FFA-only treatment group.

The lipid lowering activity and combinational design of isolated CG components. Uniform Design was employed to investigate the synergistic effect of three active components (CG-4, CG-10, CG-14) with varying proportions. Each combination group is equal in concentration $(20 \mu \mathrm{M})$. The numbers in each row indicate a volume ratio of three monomers. The lipid droplet reduction rate (compared with the model group) was used to evaluate the therapeutic effect of each component mixture group as shown in Table 2.

Response Surface Methodology (RSM). RSM is an important subject in the statistical design of experiments; it is a collection of mathematical and statistical techniques useful for modeling and analyzing problems for which a response is influenced by several variables $^{22}$. The goal is to move rapidly and efficiently along a path to obtain a maximum or a minimum response, such that the response 

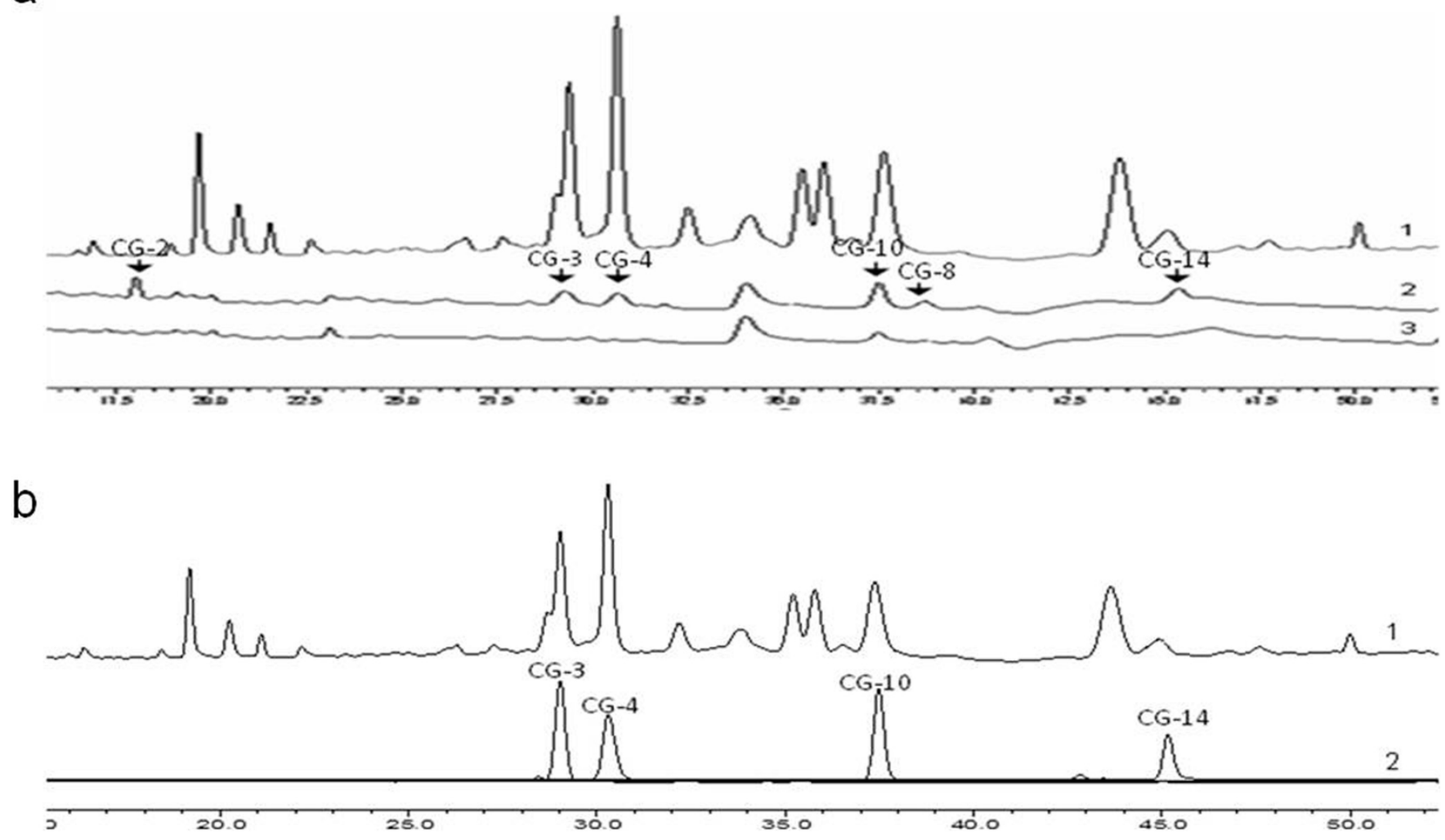

Figure 4 | HPLC-UV analysis for the serum chemistry assay. (a) Comparison of the chromatograms of (1) the CG extract, (2) the serum of high dose CG extract group, and (3) the serum of the BD group; (b) Comparison of (2) the chromatograms of CG-3, CG-4, CG-10 and CG-14 with (1) the chromatogram of CG extract. Peaks represent the intake of different mono-compounds intracellularly.

is optimized. RSM aims to find the optimal process settings to achieve peak performance. In this paper, we utilized the model $\mathrm{Y}=\mathrm{f}\left(\mathrm{X}_{1}, \mathrm{X}_{2}, \mathrm{X}_{3}\right)+\mathrm{e}$.

The variables are independent variables, such that the response depends on them. The experimental error term, denoted as e, represents any measurement error on the response, as well as other types of variations not considered. The Least Square method was used to estimate the parameters in the polynomials. We used the Design Expert 8.0.5b to complete the above computation process. The results are shown in Figure 9. Individual treatment with components CG-4, CG-10 and CG-14 (group 9, 10 and 11 from Table 2) cannot decrease the lipid content as effectively as the three in combination. From the mathematic model, we can get a predicted best lipid reduction rate of $31.13 \%$ by combination of CG-4, CG-10 and CG-14 (CG-4: CG-10: CG-14 = 2.065:1.782:2.153) rather than monocompounds.

\section{Discussion}

In this study, we attempted to test the lipid-lowering effects of CG extract on different fat-overload models. The lipid-lowering and hepatoprotective activities of CG extract were confirmed in the FFA-induced HepG2 cell model (Fig. 2), the AAP-induced L02 cell model (Fig. 3) and the HFD model for NAFLD in male SD rats (Fig. 1). The results of biochemistry indicators (Table 1), pathological observation (Fig. 1) and cell morphology (Fig. 2, Fig. 3) analysis suggested that CG extract could decrease the amount of serum and cellular lipid while protecting the cells from inflammation and injury. Mono-compounds from the CG-extract were isolated and found to be effective on multiple targets within hepatocytes (Fig. 8).

As a traditional Chinese medicine, CG extract is proven to be an effective drug in curing hepatic disease, but its component range and working mechanism remain unknown. We used serum pharmaco- chemical approaches (Fig. 4) to discover useful components in CG extract and optimized their proportions to enhance its therapeutic effect.

TCM serum pharmaco-chemistry, since Japanese scientist Oguri $\mathrm{T}^{23}$ first introduced its concept in the mid-1980s, has attracted growing attention from Chinese researchers. This theory proposes that most herbs are transformed into complex chemical constituents

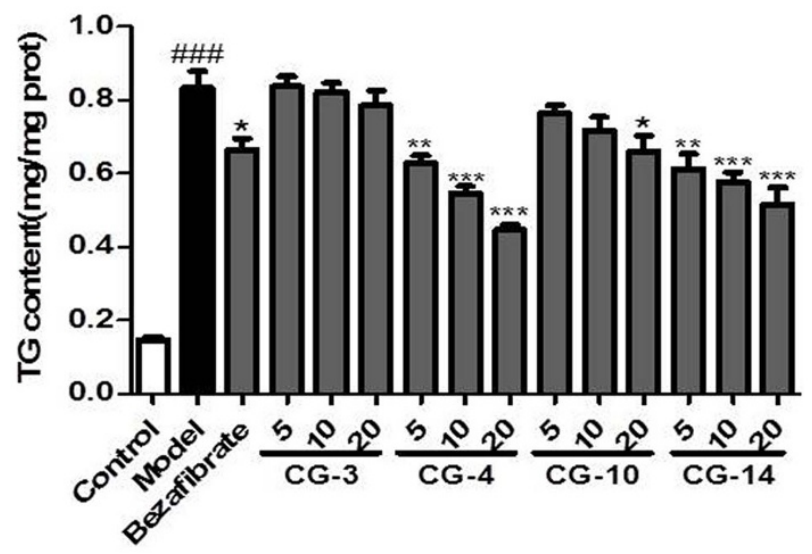

Figure $5 \mid$ Measurement of Triglycerides (TG) in FFA-induced lipid storage within HepG2 cells. HepG2 cells were cultured for 24 hours and induced with FFA (OA/PA) and/or mono-compounds from CG (CG-3, CG-4, CG-10, CG-14). The concentration used for positive drug was $20 \mu \mathrm{M}$, and the concentrations used for mono-compounds were at 5, 10 and $20 \mu \mathrm{M}$. The values are shown as the means \pm s.d. and are representative of three independent experiments. ${ }^{* *}$ for $\mathrm{p}<0.01 ; * *$ for $\mathrm{p}<0.001$ vs model and ${ }^{\# \#}$ symbolizes $\mathrm{p}<0.001$ vs control. 
a
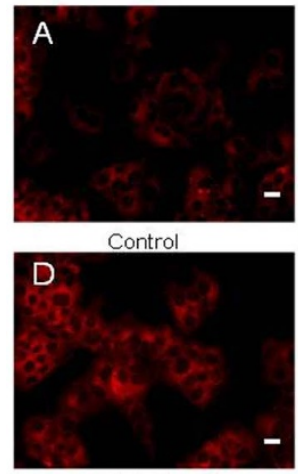

CG-4 $20 \mu \mathrm{M}$
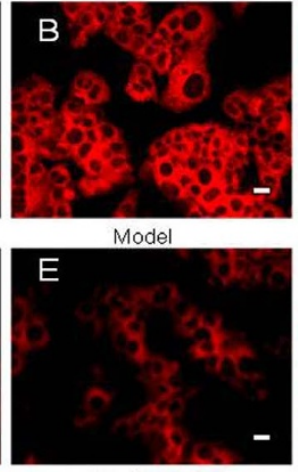

CG-10 $20 \mu \mathrm{M}$

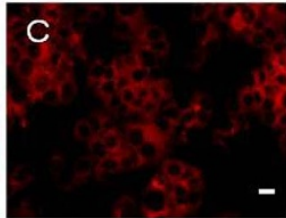

Bezafibrate $20 \mu \mathrm{M}$

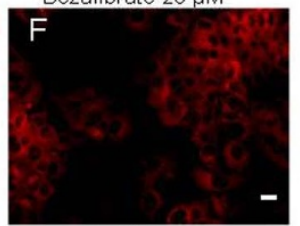

CG-14 $20 \mu \mathrm{M}$ b

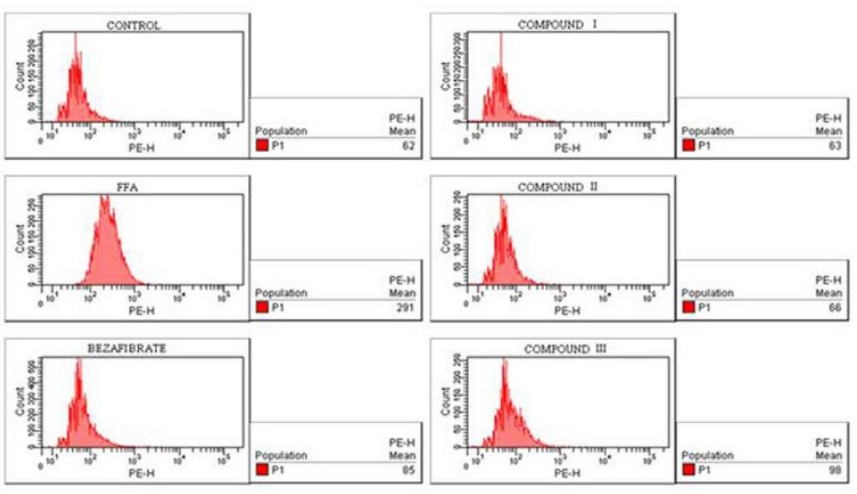

Figure 6 Flow cytometry method for the lipid droplet determination of FFA induced HepG2 cells administered with or without Bezafibrate and CG-4, CG-10, CG-14 (scale bars $=50 \mu \mathrm{m}$ ). (a) Nile red staining for the morphological observation of the lipid contents in the HepG2 cell. (A) Control; (B) FFAinduced HepG2 cell model group; (C) FFA-induced HepG2 cell model administered with Bezafibrate $(20 \mu \mathrm{M})$; (D-F) 1 mM FFA-induced HepG2 cell high fat model affected by CG-4, CG-10, CG-14 (20 $\mathrm{MM})$. (b) Flow cytometry method for lipid content determination of the $1 \mathrm{mM}$ FFA-induced HepG2 cell model given Bezafibrate and CG-4, CG-10, CG-14. The molecular weight for Bezafibrate, CG-4 (Compound I), CG-10 (Coumpound II), CG-14 (Coumpound III) was $20 \mu \mathrm{M}$.

during herb preparation and a series of effects occurs after oral administration; only parts of the compounds are selectively absorbed into the body and affect target tissues. Therefore, the hypothesis that chemical constituents in the blood are the truly effective substances has been suggested. Hence, we used the highly effective liquid chromatography (HPLC) technology for the study of serum pharmacochemistry of CG extract. The comparison of HPLC fingerprints among serum samples and the CG extract revealed that $6 \mathrm{CG}$ constituents enter the blood. CG-2 and CG- 8 may be the metabolites, while CG-3, CG-4, CG-10, CG-14 are of CG origin. Following invitro drug screening experiments showed that CG-4, CG-10 and CG14 are the most active constituents and are therefore considered to be the core factors in the reduction of hepatic lipids.

After we found that CG mono-compounds CG-4, CG-10 and CG14 posses lipid-lowering and hepatoprotective activities, we continued our study on key lipid targets (like CD36, FATP5 and PPAR- $\alpha$ ) at the molecular level to see if the CG-extract exhibits multi-target activity on lipid oxidation and lipid absorption pathways (Fig. 4). The membrane glycoprotein CD36 plays an important role in mediating lipid uptake and is related to the pathogenesis of several metabolic disorders, such as diabetes, obesity, and nonalcoholic hep- atic steatosis ${ }^{24,25}$. The role of CD 36 in metabolic disease includes modulating intracellular lipid accumulation, lipotoxicity and insulin resistance ${ }^{26}$. Fatty acid transport proteins (FATPs) are integral transmembrane proteins that enhance the cellular uptake of long-chain and very long-chain fatty acids ${ }^{27}$. FATPs comprise a family of six highly homologous proteins, FATP1 through FATP6, which are found in all fatty acid-utilizing tissues of the body. FATP5 expression is entirely liver specific and plays a key role in lipid uptake and hence lipid storage ${ }^{28}$. CG-4, CG-10 and CG-14 attenuated the expression of both CD36 and FATP5. This suggests that these compounds influence lipid uptake mechanisms as part of their lipid-lowering capacity. Moreover, peroxisome proliferator-activated receptor- $\alpha$ $(\mathrm{PPAR}-\alpha)$ is a transcription factor and a major regulator of lipid metabolism in the liver ${ }^{29}$. The activation of PPAR- $\alpha$ promotes the uptake, utilization, and catabolism of fatty acids through the upregulation of genes involved in fatty acid transport and peroxisomal and mitochondrial fatty acid $\beta$-oxidation ${ }^{30}$. CG-4, CG-10 and CG-14 increase the protein expression of PPAR- $\alpha$ and point towards inner targets of these compounds. We thus hypothesize here that two possible mechanisms responsible for the ability of the CG components to regulate lipid metabolism in the liver are likely. One pos-<smiles>O=C(O)[C@H]1O[C@@H](Oc2c(-c3ccc(O)c(O)c3)oc3cc(O)cc(O)c3c2=O)[C@H](O)[C@@H](O)[C@H]1O</smiles>

CG-4<smiles>O=c1c(O[C@@H]2O[C@H](CO)[C@@H](O)[C@H](O)[C@H]2O)c(-c2ccc(O)c(O)c2)oc2cc(O)cc(O)c12</smiles>

CG-10 isoquercitrin
C<smiles>O=c1c(O)c(-c2ccc(O)c(O)c2)oc2cc(O)cc(O)c12</smiles>

CG-14

quercetin

Figure 7 | Chemical structures of CG-4, CG-10 and CG-14. (A) quercetin-3-O- $\beta$-D-glucuronide, (B) isoquercitrin, and (C) quercetin. 
a

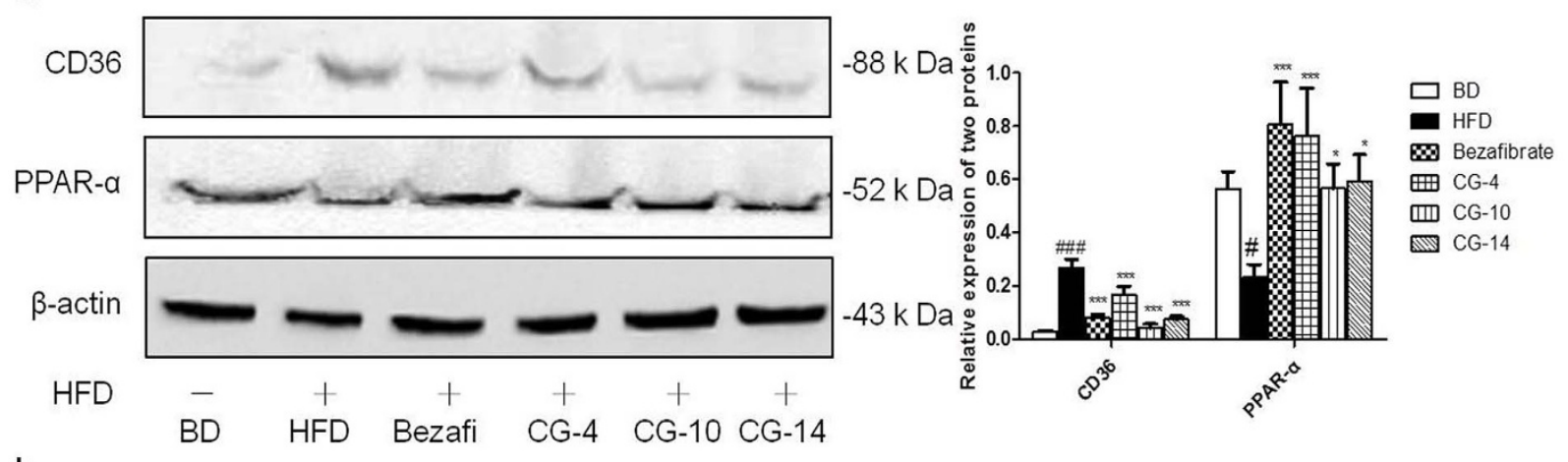

b
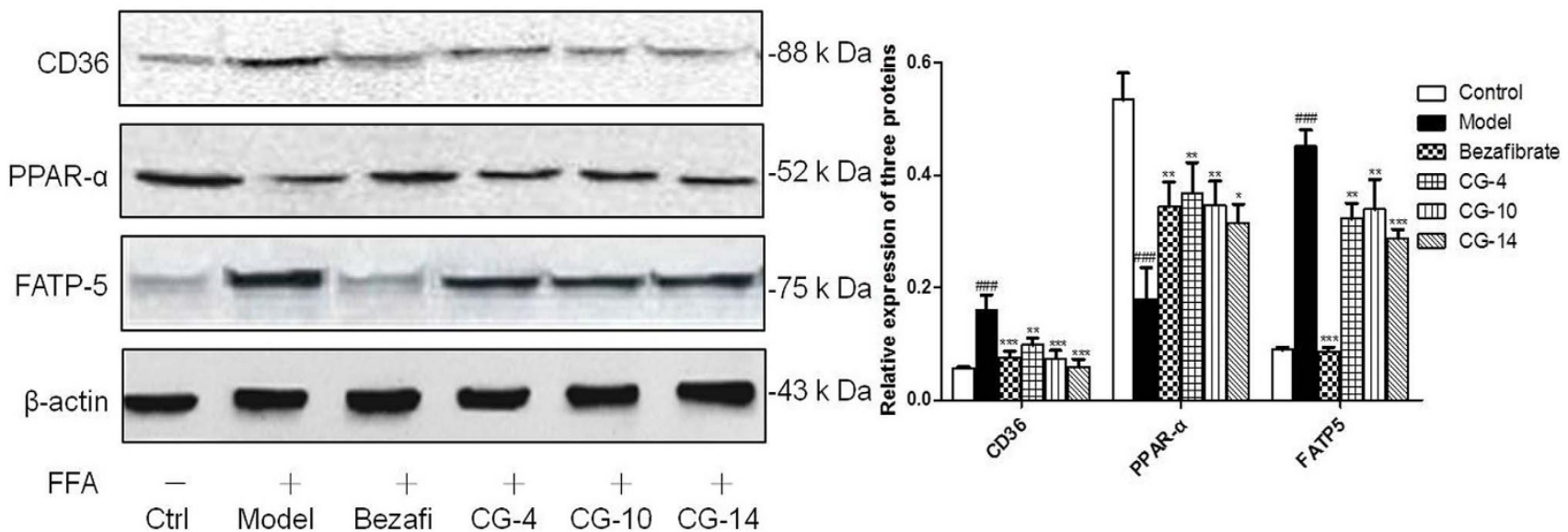

Figure 8 The effects of different CG mono-compounds and Bezafibrate on different downstream lipid regulatory genes. (a) Liver tissue; (b) HepG2 cells. Results were normalized to $\beta$-actin. As shown in Fig. 8, mono-compounds CG-4, CG-10, and CG-14 up-regulated the expression of PPAR- $\alpha$ and down-regulated the gene expression of CD36 and FATP5. The values are shown as the means \pm s.d. and are representative of three independent

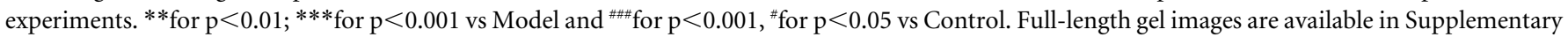
Figure 1.

sibility is that the CG mono-compounds might inhibit FFA transport and absorption. Another possibility is that the compounds increase lipid utilization, including lipid $\beta$-oxidation. This opens a wide new area of research on these compounds, which are likely to affect multiple targets in the complex disease of NAFLD. Further research on these compounds will be helpful in devising long-sought-after treatment options for NAFLD.

To optimize the proportion of the three mono-compounds to exert the maximum pharmacological effect, we adopted the math-

Table 2 | Combinations in Uniform Design and their lipid droplet reduction rate (mean \pm s.d.)

\begin{tabular}{lcccc} 
GROUP & CG-4 & CG-10 & CG-14 & LDs (\%) \\
\hline 1 & 1 & 3 & 2 & $24.95 \pm 3.17$ \\
2 & 2 & 1 & 3 & $27.18 \pm 5.60$ \\
3 & 2 & 2 & 2 & $31.05 \pm 5.34$ \\
4 & 3 & 1 & 2 & $23.44 \pm 3.74$ \\
5 & 1 & 2 & 3 & $16.92 \pm 3.48$ \\
6 & 2 & 3 & 1 & $19.85 \pm 2.23$ \\
7 & 3 & 2 & 1 & $17.61 \pm 3.88$ \\
8 & 4 & 1 & 1 & $15.72 \pm 4.77$ \\
9 & 6 & 0 & 0 & $14.00 \pm 5.92$ \\
10 & 0 & 6 & 0 & $13.63 \pm 5.33$ \\
11 & 0 & 0 & 6 & $9.30 \pm 5.03$ \\
\hline
\end{tabular}

Lipid droplet content was measured by the flow cytometer method, as mentioned above, and the data were transformed into a reduction rate (\%) compared with FFA treatment model group. ematical model called the Uniform Design and RSM method (Fig. 9). The best lipid reduction rate (of $31.13 \%$ ) is achieved with a CG-4: CG-10: CG-14 ratio of 2.065:1.782:2.153. We can thus assume that mixtures might cumulatively produce higher effects. However, additional work is needed to validate our current result and we will continue to use the mathematical model to balance the varied positive and negative effects with the overall disease curing purpose.

Consequently, our work confirmed that CG extract has a lipid lowering capacity both in vitro and in vivo. We also confirmed and indicated that the mono-compounds may be responsible for the CG extract lipids reducing activity. We also showed that mono-compounds CG-4, CG-10, CG-14 have lipid-lowering effects and that these effects may be manifested through multitargets to achieve hepatoprotective activities. We used a combination of pharmacological and statistical techniques to form a suitable model for developing drug combinations. Furthermore, we have indicated the best combination of mono-compounds present in CG extract that is responsible for lipid-lowering activity. Hence, this study forms the basis for future research on these pharmacologically active compounds and confirms their effectiveness. This study also uses methodologies that can be helpful in the future modernization of TCMs during modern drug development procedures.

\section{Methods}

Collection of plant material and preparation of plant extract. The CG plant, purchased from Xinjiang DeHong Biotechnology Corporation was dried and grounded into a fine powder. The CG powder was extracted in $70 \%$ ethanol 
a

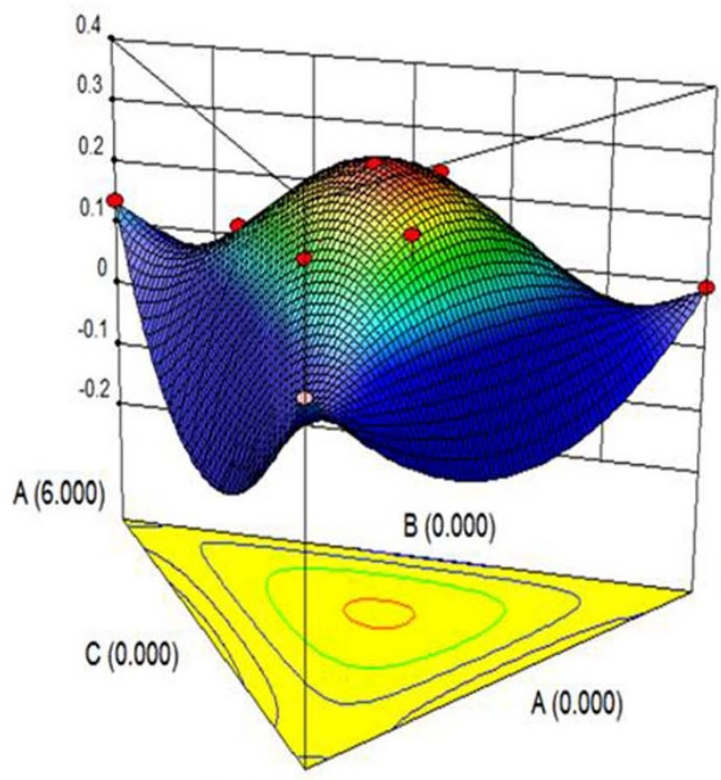

$B(6.000)$ b

$C(6.000)$

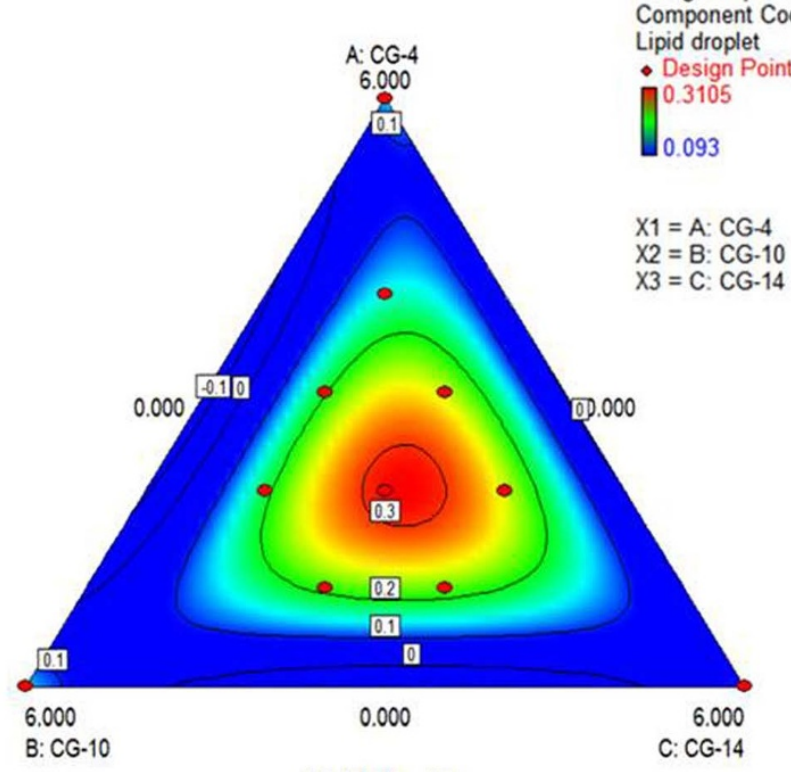

Lipid droplet

Figure 9 Response surface methodology and contour plots of lipid droplet reduction effect for component combinations. (a) 3D response surface of the lipid droplet reduction rate (\%); (b) contour plots of the lipid droplet reduction rate (\%). The results of this figure were predicted by the data reported in table 2 .

$(1: 16, \mathrm{w} / \mathrm{v})$ for $2 \mathrm{~h}$ at room temperature $\left(24^{\circ} \mathrm{C}\right)$ with sonic extraction. The ethanol extract was filtered, and the solvent was removed under vacuum, using a rotary evaporator.

Chemicals. Bezafibrate was provided by Tasly Pharmaceutical Co. Ltd. Four CG compounds (CG-3, CG-4, CG-10, CG-14) were extracted and separated in a pharmaco-chemistry laboratory at the China Pharmaceutical University using the method described in our previous study ${ }^{21}$.

Animal treatment. Two completely independent animal experiments were conducted as follows:

1. The first animal experiment for serum and liver biochemical analysis, liver histopathological analysis and serum chemistry study. Thirty male Sprague-Dawley (SD) rats weighing 180 200 g at 5-6 weeks of age were purchased from the Qinglongshan Animal Laboratory Center (Shanghai, China). The animals were allowed free access to food and tap water throughout the acclimatization and experimental periods. The animals were exposed to 12 hours of light/dark cycle during the study. This study was approved by the Ethical Committee of China Pharmaceutical University, Nanjing University, and the Laboratory Animal Management Committee of Jiangsu Province (Approval No.:20090002). All animal experiments were conducted in compliance with the standard ethical guidelines under the control of the ethical committees mentioned above. After acclimatization for one week, the rats were randomly divided into five experimental treatment groups $(\mathrm{n}=6$ mice each) as follows: (a) Basal diet (BD) group: fed with a normal diet (b) High fat diet (HFD) group; fed with a high caloric diet model (c) Bezafibrate treatment group: fed with HFD and given Bezafibrate (100 $\mathrm{mg} / \mathrm{kg} /$ day) (d) CG extract low dose treatment group (4.8 $\mathrm{g} / \mathrm{kg} /$ day): fed with HFD and given CG extract. (e) CG extract high dose treatment group (9.6 g/ $\mathrm{kg} /$ day) fed with high HFD and given CG extract. All five groups received their respective treatments for 9 weeks. The Bezafibrate and CG extract were suspended in a $0.5 \%$ carboxymethylcellulose solution and was administered by oral gavage once per day. Rats in control group were given the $0.5 \%$ carboxymethylcellulose solution only. The body weights of individual rats were monitored weekly.

2. Animal experiment for western blotting study. Thirty-six male Sprague-Dawley (SD) rats weighing 180 200 g at 5-6 weeks of age were randomly divided into six experimental treatment groups $(\mathrm{n}=6$ rats each) after acclimatization for one week as follows: (a) Basal diet (BD) group: fed with a normal diet (b) High fat diet (HFD) group; fed with high caloric diet model (c) Bezafibrate treatment group: fed with HFD and given Bezafibrate (100 mg/kg/day) (d) CG-4 treatment group (100 mg/kg/day): fed with HFD and given CG-4. (e) CG-10 treatment group (100 mg/kg/day) fed with high HFD and given CG-10. (f) CG-14 treatment group ( $100 \mathrm{mg} / \mathrm{kg} / \mathrm{day})$ fed with high HFD and given CG-14. All animal care and experimental procedures were carried out in accordance with the methods mentioned above.
The basal diet and high fat diet were provided by the Jiangsu Xietong Medical and Biological Corporation (Nanjing, China). The HFD contains $506.8 \mathrm{kcal} / 100 \mathrm{~g}$ (lard, $10 \mathrm{~g} / 100 \mathrm{~g}$; bile salt, $0.5 \mathrm{~g} / 100 \mathrm{~g}$; cholesterol, $1 \mathrm{~g} / 100 \mathrm{~g}$; powdered milk $5 \mathrm{~g} / 100 \mathrm{~g}$; egg yolk powder $3 \mathrm{~g} / 100 \mathrm{~g}$; sugar $5 \mathrm{~g} / 100 \mathrm{~g}$; methylthiouracil $0.2 \mathrm{~g} / 100 \mathrm{~g}$; basal diet $75.3 \mathrm{~g} / 100 \mathrm{~g})$. The basal diet contains $360 \mathrm{kcal} / 100 \mathrm{~g}(13.3 \mathrm{~g} / 100 \mathrm{~g}$ from fat, $26.2 \mathrm{~g} /$ $100 \mathrm{~g}$ from protein, and $60.5 \mathrm{~g} / 100 \mathrm{~g}$ from carbohydrate).

Serum and liver biochemical analysis. After the completion of the study, all rats were sacrificed at once and serum was withdrawn instantaneously after blood collection by centrifugation at $3500 \mathrm{rpm}$ for 5 minutes. The serum ALT and AST were determined using enzymatic colorimetric methods. The serum lipid contents: Total cholesterol (TC), Total triglycerides (TG), Low-density lipoprotein-cholesterol (LDL-C), and High-density lipoprotein-cholesterol (HDL-C) were investigated to evaluate the relative lipid content changes. Hepatic levels for MDA and LDH were also analyzed. Protein concentration was measured by bicinchoninic acid assay (BCA), and values were normalized according to the protein concentration. All above-mentioned parameters were measured by commercially available kits provided by the Nanjing Jiancheng Bioengineering Institute, Nanjing, China.

Liver histopathological analysis. Histopathological analysis was performed by incising standardized specimen from specified portions of liver. Briefly, liver tissues were fixed in $10 \%$ formalin for 4 hours, and dehydrated in a graded series of ethanol and embedded in paraffin wax. 4 - $\mu \mathrm{m}$-thick sections were cut and stained with hematoxylin-eosin (H\&E) staining and analyzed under Olympus-BX53 biological microscope. To evaluate fat deposition, fresh liver samples were embedded using tissue OCT-freeze medium and sliced into $8-10 \mu \mathrm{m}$-thick sections using a cryostat microtome (LEICA CM1850). The frozen sections were then stained with Oil red O for 15 minutes before being analyzed under an Olympus-BX53 biological microscope.

Cell culture and treatment. HepG2, a human hepatoma cell line with well differentiated morphology and hepatic function $\mathrm{s}^{31}$, was obtained from the Chinese Academy of Sciences (Shanghai, China); while the hepatocyte cell line L02, originating histologically from normal human liver tissue ${ }^{32}$, was purchased from Nanjing KeyGen Biotech Co Ltd (Nanjing, China). Both cell lines were cultured in DMEM medium (Gibco, Grant Island, USA) and supplemented with $10 \%$ heatinactivated fetal bovine serum (FBS), 100 units/ml penicillin and $100 \mathrm{mg} / \mathrm{ml}$ streptomycin at $37^{\circ} \mathrm{C}$ in a humidified incubator containing $5 \% \mathrm{CO}_{2}$.

HepG2 cells were cultured in six-well plates at the density of $1 \times 10^{6}$ for 24 hours before treating different groups with or without $1 \mathrm{mM}$ free fatty acids (FFA) for 24 hours to induce fat deposition model. There were a total of five groups (the control group; the FFA-induced group; the FFA and Bezafibrate $(20 \mu \mathrm{M})$ group; the FFA and high dose CG extract $(0.2 \mathrm{mg} / \mathrm{mL})$ group; the FFA and low dose CG extract $(0.1 \mathrm{mg} /$ $\mathrm{mL}$ ) group). To examine the lipid-lowering effect of CG extract, the treated HepG2 
cells were fixed with $4 \%$ buffered paraformaldehyde and stained with Oil red O (Sigma-Aldrich, St Louis, USA).

L02 cells were treated with or without $5 \mathrm{mmol} / \mathrm{L}$ Acetaminophen (AAP) to induce hepatotoxicity. A concentration of $0.2 \mathrm{mg} / \mathrm{mL}$ of the positive drug Diammonium glycyrrihizinate was used to compare the pharmacological activity of CG extract.

FFA treatment. We used a FFA fat-overloading model, mixing oleate and palmitate (Sigma-Aldrich, St Louis, USA) in a 2:1 ratio, to establish lipid accumulation model to induce hepatocytes as mentioned elsewhere ${ }^{33,34}$. This model has been shown to mimic the NAFLD characteristics with low level of toxicity to the cells ${ }^{35}$. Briefly, stock solutions of oleate $(1000 \mathrm{mM})$ and palmitate $(1000 \mathrm{mM})$ were prepared in dimethyl sulfoxide (DMSO) and heat-dissolved. The required FFA concentration was then added to cell culture medium (DMEM) containing the final concentration of $1 \%$ Bovine Serum Albumin (BSA). BSA was used to adopt the physiological ratio of FFABSA binding ${ }^{36}$. FFA-BSA mixture was then added to HepG2 cells at indicated times. Control groups in all cases were incubated with $1 \%$ fatty acid-free BSA without palmitate acid and oleic acid.

HPLC-UV method for serum chemistry study. We established stable HPLC-UV chromatograph condition for detecting CG extract in animal serum. A Diamonsil $\mathrm{C}_{18}$ analytical column $(250 \mathrm{~mm} \times 4.6 \mathrm{~mm}, 5 \mu \mathrm{m})$ was used with a mobile phase consisting of methanol and $0.1 \%$ acetic acid. The detecting wave-length was set at $280 \mathrm{~nm}$. Flow rate was kept at $0.8 \mathrm{~mL} / \mathrm{min}$. The column was maintained at $40^{\circ} \mathrm{C}$ and the injection volume was $20 \mu \mathrm{L}$.

In vitro drug screening. HepG2 cells were cultured for 24 hours and induced with FFA (OA/PA) and/or CG mono-compounds (CG-3, CG-4, CG-10, CG-14). The concentration for positive drug was $20 \mu \mathrm{M}$ and the concentrations for monocompounds were at 5, 10 and $20 \mu \mathrm{M}$. TG was measured by commercially available kits (Nanjing Jiancheng Bioengineering Institute, Nanjing, China), and proteins were normalized in each group after BCA. Nile red is a selective fluorescent stain for intracellular lipid droplets. Cells, fixed with $4 \%$ buffered paraformaldehyde for $15 \mathrm{~min}$, were washed with $1 \mathrm{~mL}$ PBS and then stained with Nile red (Sigma-Aldrich); Stock solution $(0.5 \mathrm{mg} / \mathrm{mL}$ in DMSO) was diluted $1: 5000$ in PBS; $500 \mu \mathrm{L}$ of this diluted Nile red solution was added into each well (six well plate) and protected from light at room temperature for $5 \mathrm{~min}$ and then washed with $1 \mathrm{~mL}$ PBS. Pictures were taken with the Olympus-BX53 biological microscope. For the quantitative analysis, HepG2 cells treated with different group of drugs were harvested, and incubated with Nile red $(1 \mu \mathrm{g} / \mathrm{ml})$ in dark for $5 \mathrm{~min}$ at room temperature. The fluorescence intensity of each sample was measured immediately by flow cytometry at an excitation wavelength of $488 \mathrm{~nm}$ and emission wavelength of $550 \mathrm{~nm}$.

Western Blotting Analysis. The cells and tissue were lysed in a radioimmunoprecipitation assay (RIPA) buffer containing protease inhibitor mixtures and phosphatase inhibitors (Beyotime Institute of Biotechnology, China). Homogenates were centrifuged at $10,000 \times g$ for $10 \mathrm{~min}\left(4^{\circ} \mathrm{C}\right)$, and the subsequent supernatants were extracted for Western blot analyses. Membranes were then incubated with the primary antibodies for FATP5 (1:1000, Abcam, USA), PPAR- $\alpha$ ( $1: 500$, Abcam, USA), CD36 ( $1: 2000$, Abcam, USA), followed by incubation with a secondary antibody $(1: 4000$, Santa Cruz Biotechnology, USA). Antibody expression was viewed using enhanced chemiluminescence reagents (PerkinElmer, USA). Densitometric scanning of band intensities were quantified by Quantity One (BioRad, USA). $\beta$-actin ( $1: 500$, Santa Cruz Biotechnology, USA) was used as an internal control.

Drug combination. To further discover the synergistic effect of the monocompounds, a Uniform Design was employed to arrange the experiment. The details regarding Uniform Design are described in many references ${ }^{37-39}$. A Response Surface Methodology (RSM) method was used to optimize the combination of CG-4, CG-10 and CG-14.We used the flow cytometer method to determine the intracellular lipid content of different combination groups.

Statistical analysis. The data are represented as the means \pm s.d. The significance of the difference between groups was evaluated with a one-way analysis of variance (ANOVA) and Dunnett's t-test. Statistical differences were considered significant when $\mathrm{p}<0.05$

1. Naik, A., Košir, R. \& Rozman, D. Genomic aspects of NAFLD pathogenesis. Genomics. 102, 84-95 (2013).

2. Fon Tacer, K. \& Rozman, D. Nonalcoholic fatty liver disease: focus on lipoprotein 691 and lipid deregulation. J. Lipids. 2011, 14(2011).

3. Gregor, L. \& Damjana, R. Cholesterol and inflammation at the crossroads of nonalcoholic fatty liver disease (NAFLD) and atherogenesis. Atherogenesis. Chapter 13, 281-305 (2012)

4. Elizabeth, M. B. \& Dina, G. T. Histopathology of nonalcoholic fatty liver disease. World J. Gastroenterol. 16, 5286-5296(2010).

5. Brunt, E. M. Nonalcoholic steatohepatitis. Semin. Liver Dis. 24, 3-20 (2004).

6. Kallwitz, E. R., McLachlan, A. \& Cotler, S. J. Role of peroxisome proliferatorsactivated receptors in the pathogenesis and treatment of nonalcoholic fatty liver disease. World J. Gastroenterol. 14, 22-28 (2008).
7. Doege, H. et al. Targeted deletion of FATP5 reveals multiple functions in liver metabolism: alterations in hepatic lipidhomeostasis. Gastroenterology 130, 1245-1258 (2006).

8. Buqué, X. et al. High insulin levels are required for FAT/CD36 plasma membrane translocation and enhanced fatty acid uptake in obese Zucker rat hepatocytes. Am. J. Physiol. Endocrinol. Metab. 303, 504-514 (2012).

9. Editorial board of Flora Republicae Popularis Sinicae of Chinese Academy of Sciences. Flora Repubulicae Popularis Sinicae (Science Press, Beijing, 1993).

10. China Pharmacopoeia Committee. Pharmacopoeia of the People's Republic of China. 1th division of 2010 edition (Chemical Industry Press, Beijing, 2010).

11. Yao, X., Zhu, L., Chen, Y., Tian, J. \& Wang, Y. In vivo and in vitro antioxidant activity and $\alpha$-glucosidase, $\alpha$-amylase inhibitory effects of flavonoids from Cichorium glandulosum seeds. Food Chem. 139, 59-66 (2013).

12. Upur, H., Amat, N., Blazeković, B. \& Talip, A. Protective effect of Cichorium glandulosum root extract on carbon tetrachloride-induced and galactosamineinduced hepatotoxicity in mice. Food Chem. Toxicol. 47, 2022-2030 (2009).

13. Wu, H., Su, Z., Yang, Y., Ba, H. \& Aisa, H. A. Isolation of three sesquiterpene lactones from the roots of Cichorium glandulosum Boiss. et Huet. by high-speed countercurrent chromatography. J. Chromatogr A. 1176, 217-222 (2007).

14. Wu, H. K. et al. Isolation of esculetin from Cichorium glandulosum by high speed countercurrent chromatography. Chem. Nat. Compd. 43, 109 (2007).

15. Yoon, B. J. Enhanced stochastic optimization algorithm for finding effective multi-target therapeutics [J]. BMC Bioinformatics.12, Suppl 1, S18 (2011).

16. Wong, P. K. et al. Closed-loop control of cellular functions using combinatory drugs guided by a stochastic search algorithm. Proc. Natl Acad. Sci. USA 105, 5105-5110 (2008).

17. Wu, X. et al. Prevention of free fatty acid-induced hepatic lipotoxicity by 18 betaglycyrrhetinic acid through lysosomal and mitochondrial pathways. Hepatology 47, 1905-1915 (2008).

18. Sanz-Garcia, C. et al. Sterile inflammation in acetaminophen-induced liver injury is mediated by Cot/tpl2. J. Biol. Chem. 288, 15342-15351 (2013).

19. Bajt, M. L., Knight, T. R., Lemasters, J. J. \& Jaeschke, H. Acetaminophen-induced oxidant stress and cell injury in cultured mouse hepatocytes: protection by Nacetyl cysteine. Toxicol. Sci. 80, 343-349 (2004).

20. Thomson, J. S. \& Prescott, L. F. Liver damage and impaired glucose tolerance after paracetamol overdosage. Br. Med. J. 27, 506-507 (1966).

21. Yang, W. Z., Wang, H., Zhang, J., Feng, F. \& Xie, N. Chemical constituents from Cichorium glandulosum. Chinese Journal of Natural Medicines 3, 193-195 (2009).

22. Douglas, C. Montgomery. Design and analysis of experiments. 7th edition (Wiley, New York, 2007).

23. Oguri, T. Clinical and bacteriological studies of Streptococcus pneumoniae Yearly changes in isolation frequencies from clinical specimens, serotype distributions and drug susceptibilities, especially those of beta-lactam-resistant strains. Jpn. J. Antibiot 39, 783-806 (1986).

24. Alkhatatbeh, M. J., Enjeti, A. K., Acharya, S., Thorne, R. F. \& Lincz, L. F. The origin of circulating CD36 in type 2 diabetes. Nutr. Diabetes 3, e59 (2013).

25. Le Foll, C., Dunn-Meynell, A., Musatov, S., Magnan, C. \& Levin, B. E. FAT/CD36: A major regulator of neuronal fatty acid sensing and energy homeostasis in rats and mice. Diabetes 62, 2709-2716 (2013).

26. He, J., Lee, J. H., Febbraio, M. \& Xie, W. The emerging roles of fatty acid translocase/CD36 and the aryl hydrocarbon receptor in fatty liver disease. Exp. Biol. Med. (Maywood) 236, 1116-1121 (2011).

27. Kazantzis, M. \& Stahl, A. Fatty acid transport proteins, implications in physiology and disease. Biochim. Biophys. Acta 1821, 852-857 (2012).

28. Gimeno, R. E. Fatty acid transport proteins. Curr. Opin. Lipidol. 18, 271-276 (2007).

29. Ringseis, R. \& Eder, K. Regulation of genes involved in lipid metabolism by dietary oxidized fat. Mol. Nutr Food Res. 55, 109-121 (2011).

30. Petrescu, A. D. et al. Impact of L-FABP and glucose on polyunsaturated fatty acid induction of PPAR $\alpha$-regulated $\beta$-oxidative enzymes. Am. J. Physiol. Gastrointest. Liver Physiol. 304, 241-256 (2013).

31. Mersch-Sundermann, V., Knasmüller, S., Wu, X. J., Darroudi, F. \& Kassie, F. Use of a human-derived liver cell line for the detection of cytoprotective, antigenotoxic and cogenotoxic agents. Toxicology 198, 329-340 (2004).

32. Zhang, W. Y., Cai, N., Ye, L. H. \& Zhang, X. D. Transformation of human liver LO2 cells mediated by stable HBx transfection. Acta Pharmacol Sin. 30, 1153-1161(2009).

33. Gunduz, F. et al. Free fatty acids induce ER stress and block antiviral activity of interferon alpha against hepatitis C virus in cell culture. Virol. J. 9, 143 (2012).

34. Akazawa, Y. et al. Palmitoleate attenuates palmitate-induced Bim and PUMA upregulation and hepatocyte lipoapoptosis. J. Hepatol. 52, 586-593 (2010).

35. Gomez-Lechon, M. J. et al. A human hepatocellular in vitro model to investigate steatosis. Chem. Biol. Interact. 165, 106-116 (2007).

36. Yamasaki, M., Chujo, H., Nou, S., Tachibana, H. \& Yamada, K. Alleviation of the cytotoxic activity induced by trans10, cis12-conjugated linoleic acid in rat hepatoma dRLh-84 cells by oleic or palmitoleic acid. Cancer Lett. 196, 187-196 (2003).

37. Fang, K. T., Li, F. \& Agus, S. Design and modeling for computer experiments (Chapman \& Hall/CRC, Boca Raton, 2006). 
38. Fang, K. T., Ge, G. N., Liu, M. Q. \& Qin, H. Construction of uniform designs via super-simple resolvable $t$-designs (Department of Mathematics, Hong Kong Baptist University, 2004).

39. Fang, K. T., Lu, X., Tang, Y. \& Yin, J. X. Constructions of uniform designs by using resolvable packings and coverings. Discrete Mathematics 274, 25-40 (2004).

\section{Acknowledgments}

This study was supported by "National Science and Technology Infrastructure Program of China" (2012BAI30B001), "Mega-projects of Science Research for the 12th Five Year Plan of China" (2011ZX09401007), "Project Program of State Key Laboratory of Natural Medicines, China Pharmaceutical University(JKGZ201108) and "Jiangsu Yanjiushengchuangxin Plan"(CXLX11-0782).

\section{Author contributions}

L.D. designed and performed experiments, analyzed data and wrote the manuscript. J.L. conducted the mathematical experiments and analyzed data. W.H. and L.W. helped with experimental design, data analysis and manuscript revision. F.Y. and J.S. are responsible for the experimental design, data coordination, analysis and interpretation, writing, revising and finalization of the manuscript. F.Y. and J.S. are also responsible for the decision to submit the manuscript for publication. All authors read and approved the final manuscript.

\section{Additional information}

Supplementary information accompanies this paper at http://www.nature.com/ scientificreports

Competing financial interests: The authors declare no competing financial interests

How to cite this article: Ding, L. et al. Lipid modulatory activities of Cichorium glandulosum Boiss et Huet are mediated by multiple components within hepatocytes. Sci. Rep. 4, 4715; DOI:10.1038/srep04715 (2014).

(c) (1) (-) This work is licensed under a Creative Commons Attribution-NonCommercialNoDerivs 3.0 Unported License. The images in this article are included in the article's Creative Commons license, unless indicated otherwise in the image credit; if the image is not included under the Creative Commons license, users will need to obtain permission from the license holder in order to reproduce the image. To view a copy of this license, visit http://creativecommons.org/licenses/by-nc-nd/3.0/ 